\title{
Single-cell RNA sequencing reveals regulation of fetal ovary development in the monkey (Macaca fascicularis)
}

\author{
Zheng-Hui Zhao ${ }^{1,2}$, Chun-Yang Li $i^{3}$, Tie-Gang Meng ${ }^{1,4}$, Yan Wang ${ }^{3}$, Wen-Bo Liư ${ }^{5}$, Ang Li', Yi-Jun Cai ${ }^{3}$, Yi Hou', \\ Heide Schatten ${ }^{6}$, Zhen-Bo Wang $\mathbb{1 0}^{1,2}$, Qing-Yuan Sun (1) ${ }^{4}$ and Qiang Sun ${ }^{3}$
}

\begin{abstract}
Germ cells are vital for reproduction and heredity. However, the mechanisms underlying female germ cell development in primates, especially in late embryonic stages, remain elusive. Here, we performed single-cell RNA sequencing of 12,471 cells from whole fetal ovaries, and explored the communications between germ cells and niche cells. We depicted the two waves of oogenesis at single-cell resolution and demonstrated that progenitor theca cells exhibit similar characteristics to Leydig cells in fetal monkey ovaries. Notably, we found that ZGLP1 displays differentially expressed patterns between mouse and monkey, which is not overlapped with NANOG in monkey germ cells, suggesting its role in meiosis entry but not in activating oogenic program in primates. Furthermore, the majority of germ cell clusters that sharply express PRDM9 and SPO11 might undergo apoptosis after cyst breakdown, leading to germ cell attrition. Overall, our work provides new insights into the molecular and cellular basis of primate fetal ovary development at single-cell resolution.
\end{abstract}

\section{Introduction}

Fetal ovary development is a well-orchestrated complex process that involves the transitions of multiple cell states and communications between germ cells and niche cells. In primates, female germ cell (FGC) development processes at embryonic stages mainly include oogonia proliferation, meiosis initiation, germ cell attrition, and primordial follicle formation, which are accompanied with dynamic chromatin repackaging and transcriptional regulation. Moreover, FGCs initiate meiosis asynchronously in a wave from anterior to posterior, which results in the heterogeneity of germ cell populations in a fetal ovary ${ }^{1}$. Somatic progenitor cells in female gonads will adopt the ovary-specific cell fate after sex determination, which

Correspondence: Qing-Yuan Sun (sunqy@gd2h.org.cn) or

Qiang Sun (qsun@ion.ac.cn)

${ }^{1}$ State Key Laboratory of Stem Cell and Reproductive Biology, Institute of Zoology, Chinese Academy of Sciences, 100101 Beijing, China

${ }^{2}$ University of Chinese Academy of Sciences, 100049 Beijing, China

Full list of author information is available at the end of the article

These authors contributed equally: Zheng-Hui Zhao, Chun-Yang Li leads to their differentiation as granulosa cells in supporting cell populations or as theca cells in steroidogenic cell populations ${ }^{2}$. Interestingly, the FGC fate mainly relies on the ovarian environments established by somatic cells rather than the sex chromosomes in germ cells ${ }^{3}$.

Despite the mass data produced in recent years on fetal ovary development ${ }^{4-6}$, several important questions regarding the key developmental events, such as two waves of oogenesis, FGC attrition, and primordial follicle formation, have not been fully demonstrated, especially in non-human primates. In addition, although the origins of germ cells and granulosa cells are well established ${ }^{2,7}$, the origin of theca cells in primates remains unknown. Furthermore, the germline-niche communications through signaling pathways for critical events during fetal ovary development are poorly integrated.

Single-cell RNA sequencing (scRNA-seq) approach can efficiently identify cell types, uncover heterogeneity, and construct developmental trajectories, which is well suited for exploring fetal ovary development. To further improve 
our understanding on the germ cell development and somatic cell differentiation, we here performed scRNAseq of fetal monkey ovarian cells, especially in late embryonic stages, through 10× Genomics Chromium platform. And a transcriptional cell atlas of all cell types in the fetal ovary was established.

\section{Results \\ Identification of the ovarian cell types using single-cell transcriptomes}

Single-cell suspensions of fetal Macaca fascicularis ovaries were individually captured and processed with the 10× Chromium system (Fig. 1a). Sequencing data from two scRNA-seq libraries derived from the two stages of ovary samples were integrated for processing and analysis. From a total of 21,036 cells, we obtained an average of 57,269 reads per cell and 1593 genes per cell (Supplementary Fig. S1a). Following quality control, 12,471 cells were retained for further analysis.

To classify the different cell populations in fetal monkey ovaries, we performed unsupervised graph clustering through Seurat package ${ }^{8}$ to group the cells into cell clusters based on the similarities in their transcriptome profiles. Overall, the 15 transcriptionally distinct clusters were classified and visualized using UMAP (Uniform Manifold Approximation and Projection) (Fig. 1b) ${ }^{9}$. Notably, the clusters of germ cells $(1,2,3,4)$, endothelial cells (14), and macrophages (15) exhibit obvious overlap between E84 and E116 ovaries, while the granulosa cell populations $(5,6,7,8,9)$ exhibit slight overlap between E84 and E116 ovaries, and many cells derived from E84 and E116 ovaries were grouped into cluster 7. In addition, the Leydig cells $(10,11,12,13)$ exhibit no overlap between E84 and E116 ovaries (Fig. 1c). The granulosa cells and Leydig cells from E84 ovary and E116 ovary are separated into different clusters, which suggests that these cells may undergo dramatic differentiation during the development from E84 to E116 ovary. To further identify the clusters, we assigned the clusters based on known cell-type marker genes (Fig. 1d). The germ cell-specific marker genes were expressed solely in clusters 1-4 (DAZL, DDX4). The clusters 5-15 are somatic cell populations (VIM), which include granulosa cells (AMHR2), Leydig cells (DLK1), endothelial cells (PECAM1), and macrophages (TYROBP). Also, the germ cell marker genes displayed developmental stage-specific expression patterns. For instance, primordial germ cell marker gene TFAP2C and pluripotency marker gene NANOG were expressed in the early mitotic FGCs, whereas STRA8 was expressed in retinoid acid (RA) signaling-responsive FGCs. The cells in cluster 3 were meiotic prophase FGCs and specifically expressed SYCE3 and SPO11. Finally, the cells in cluster 4 were oogenesis phase FGCs and clearly expressed GDF9 and FIGLA (Fig. 1d). Collectively, the sequential expressed germ cell markers in clusters 1-4, respectively, could mirror the temporal order of early FGC development. Interestingly, our results also demonstrated multiple transcriptionally distinct subpopulations within the granulosa cells (five clusters) and Leydig cells (four clusters). Additionally, the top 10 differentially expressed genes were selected across cell clusters for the identification of each cell type (Fig. 1e). Together, we have profiled the transcriptomes of all cell types in the fetal monkey ovary in the present study.

\section{Cell lineage reconstruction reveals two waves of oogenesis}

To explore the FGC development at a higher resolution, we isolated and re-clustered the germ cell populations, which were visualized using UMAP (Fig. 2a, b). To further recapitulate the trajectory of early FGC development, we constructed the germ cell lineage using Monocle2 package $^{10}$, which organized the cells along the pseudotime trajectory, with mitotic FGCs and oogenesis phase FGCs concentrated at the beginning and end of its axis, respectively (Fig. 2c).

The reconstruction of the germ cell lineage divided the germ cell lineage into three developmental windows defined by the branch points of the pseudotime trajectories (Fig. 2c), allowing us to identify transition states leading to the oogenesis. We found that two waves of oogenesis occur in the germ cell lineage at the branch points 1 and 2. Noticeably, the first wave of oogenesis (branch 1) occurs following the RA-responsive stage, whereas the second wave of oogenesis (branch 2) occurs in the late meiotic prophase (Fig. 2c). The first wave of oogenesis may contribute to the medullary follicles that are activated immediately after their assembly, while the second wave of oogenesis contributes to the cortical primordial follicles that are activated after puberty and recruited regularly under hormonal control ${ }^{11,12}$. To further determine the patterns of differentially expressed genes, we examined the stage-specific marker genes, such as SALL4, LIN28A, REC8, MSX1, SYCP1/2, and JAG1, which change dynamically over the trajectory and are involved in oogonia proliferation, meiosis initiation, meiosis progression, and primordial follicle formation, respectively (Fig. 2d and Supplementary Fig. S1b). Also, to dissect the features of various stages of early FGC development, 1991 ordering genes that expressed dynamically along pseudotime were selected and clustered. The heatmap revealed stage-specific gene expression patterns that were consistent with well-organized germline development (Fig. 2e). As expected, gene ontology (GO) analysis of the clustered ordering genes (Supplementary Data S1) identified several significantly enriched biological processes, such as "mitotic cell cycle process" and "meiosis cell cycle process". In summary, these data displayed the dramatic changes of transcriptomes during early germ cell development. 

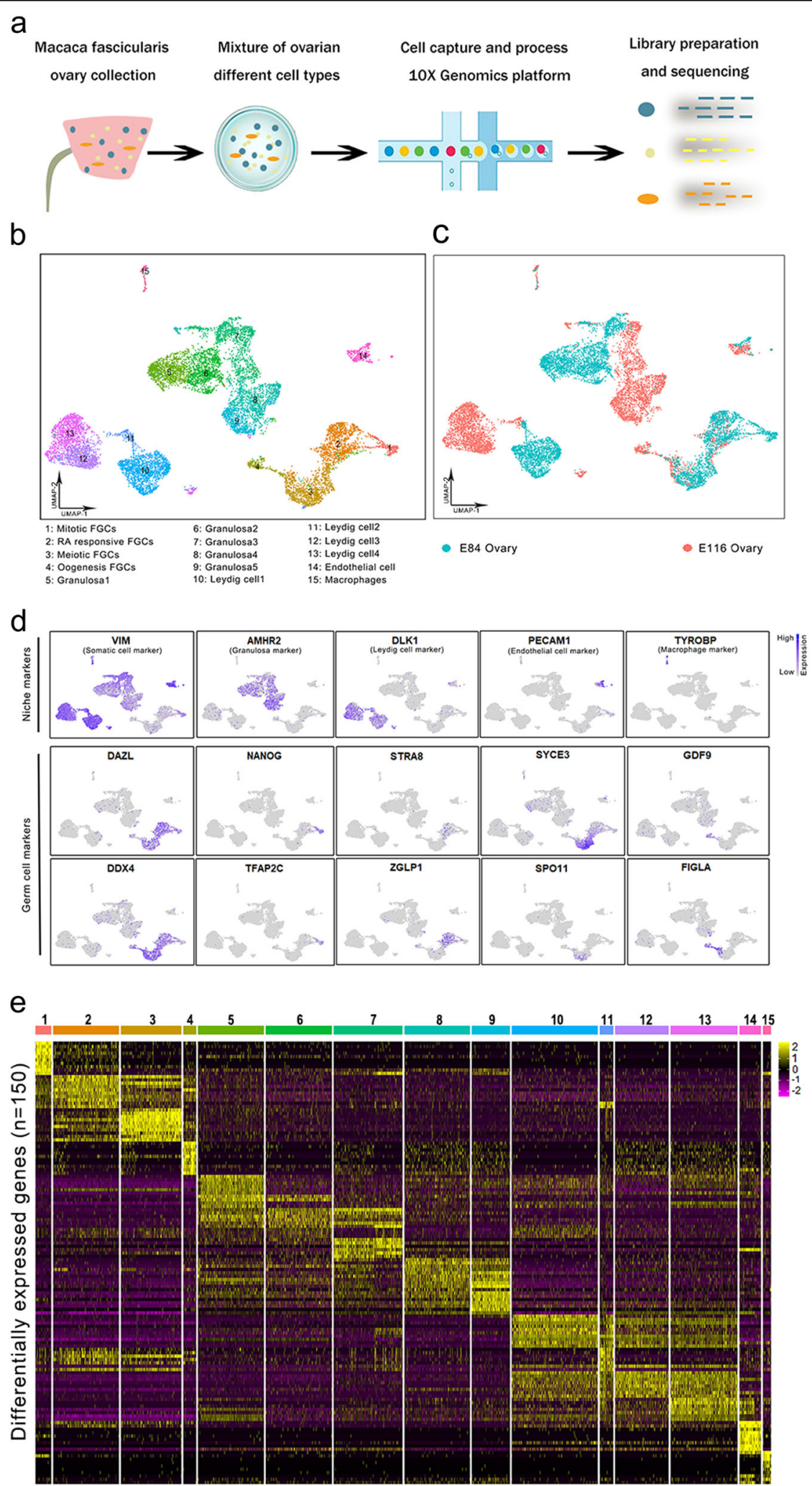

Single cells $(n=12,471)$ ordered into 15 clusters

Fig. 1 (See legend on next page.) 
(see figure on previous page)

Fig. 1 Single-cell transcriptome profiling of fetal monkey ovaries. a Schematic diagram of the experimental design. In total, fetal ovaries were collected from E84 and E116 monkey fetus and further enzymatically digested into single-cell suspension, which were then captured using 10x Genomics technology. The libraries of single-cell transcriptome were generated using the single-cell $3^{\prime}$ reagent V3 kits according to the manufacturer's protocol. b UMAP and clustering analysis of single-cell transcriptome data from fetal monkey ovaries. The 15 clusters were assigned and colored as indicated on the figure key. c UMAP plot of single-cell transcriptome data with cells colored based on their embryonic stages. d Expression patterns of selected markers for the identification of cell clusters. Blue indicates high expression and gray indicates low or no expression, as shown on the figure key. e Heatmap showing top 10 differentially expressed genes across 15 cell clusters, and 12,471 single cells were visualized.

\section{Dynamic transcription factors control meiosis initiation and progression}

Having identified the cell clusters and constructed the developmental trajectory of FGCs, we next determined the transcriptome of FGCs during their development. Notably, mitotic phase FGCs clearly expressed pluripotent markers such as NANOG and DPPA4 $4^{13,14}$ (Fig. 3a). Subsequently, the total expression levels of pluripotent genes significantly decreased during the transition from mitosis to meiosis, while meiotic marker genes, such as DMC1 and INCA1, increased strikingly (Fig. 3a). In oogenesis phase FGCs, maternal effect genes, such as ASTL and $Z A R$ 1, began to express (Fig. 3a), suggesting the start of primordial follicle formation and folliculogenesis. To further explore the transition from mitosis to meiosis, we clustered the mitotic and meiotic genes across germ cell populations. As expected, mitotic genes, such as RCC2 and $M Y B L 2$, were mainly expressed in cluster 1 , while meiotic genes, such as TEX11 and $M E I O B$, were mainly expressed in cluster 3 (Fig. 3b). Also, the CENPF, PRKDC, and $C H M P 2 A$ exhibited peak expression in RAresponsive FGCs (Fig. 3b), which may play important roles in meiosis entry.

To further dissect the differences in gene expression profiles, we detected 664, 1123, 1540, and 1070 differentially expressed genes (Supplementary Data S2) among the first four clusters, respectively. And these differentially expressed genes which changed significantly during the mitosis to meiosis transition and meiosis to oogenesis transition (Figs. 1e and 3c). Also, we performed GO analysis on the differentially expressed genes in each of the germ cell clusters (Supplementary Data S3). The genes in cluster 1 were enriched in the GO terms of "peptide metabolic process" and "stem cell population maintenance", while the genes in cluster 3 were enriched in the categories of "meiotic cell cycle" and "chromatin organization involved in meiosis", which showed striking meiosis-related features (Fig. 3d). Noticeably, RAresponsive phase FGC-specific genes were enriched in mitotic and meiotic cell cycle processes, suggesting that the cell cycle phase transition occurs at this stage.

To further explore the regulation of meiosis initiation and progression, we analyzed the differentially expressed transcription factors across germ cell clusters (Fig. 3e). We found that transcription factor genes like TEAD4,
ETV4, PRDM1, SOX15, and SOX17 showed expression peaks specifically in the mitotic stage FGCs, which may be involved in primordial germ cell proliferation. Additionally, ESX1, MSX1, RFX6, and ZGLP1 exhibited peak expression in RA-responsive stage FGCs. Evidence has shown that MSX1 and ZGLP1 are associated with meiosis initiation $^{15,16}$, but no reports exist yet about the functions of ESX1 and RFX6 in meiosis. Noticeably, DMRTC2 was specifically expressed in cluster 3 (Fig. 3e and Supplementary Fig. S1c), which might play an important role in meiosis progression ${ }^{17}$. For cluster 4 , cells showed high levels of folliculogenesis-associated genes such as FIGLA, $N O B O X$, and SOHLH1. To further compare the differences between human and monkey FGC development, we downloaded the scRNA-seq data of human $\mathrm{FGCs}^{5}$, and analyzed the expression patterns of these transcription factors (Fig. 3f). As expected, the majority of transcription factors exhibited similar patterns between human and monkey FGCs. Noticeably, both SOX15 and SOX17 were highly expressed in monkey mitotic FGCs, while only SOX15was highly expressed in human mitotic FGCs (Fig. 3e, f). Moreover, hierarchical cluster analysis of these transcription factors also showed that there was a slight difference in the expression patterns of transcription factors between human and monkey FGCs (Fig. 3e, f). In addition, the zinc-finger protein family plays important roles in early oogenesis. For example, ZNF625, ZNF217, and ZNF728 were mainly expressed in cluster 1, while ZNF131 and ZNF711 exhibited peak expression in cluster 3 (Supplementary Fig. S1d). Overall, our data demonstrate that the different germ cell clusters may represent different cell stages en route to early oogenesis.

\section{Majority of FGCs adopt an apoptotic fate}

In contrast to the second wave of oogenesis, the majority of FGCs may undergo apoptosis after the branch point 2 (Fig. 2c). To dissect the mechanisms of germ cell fate determination, we performed re-clustering of the germ cells at a higher resolution and reconstructed the germ cell lineage using Monocle2. As a result, 11 subclusters were identified and the G8 and G9 may likely undergo apoptosis, while the G7 and G10 may generate primordial follicles (Fig. 4a, b). After entering meiosis, primordial germ cell-associated genes, such as TFAP2C, were down-regulated, while meiotic genes, such as 
a

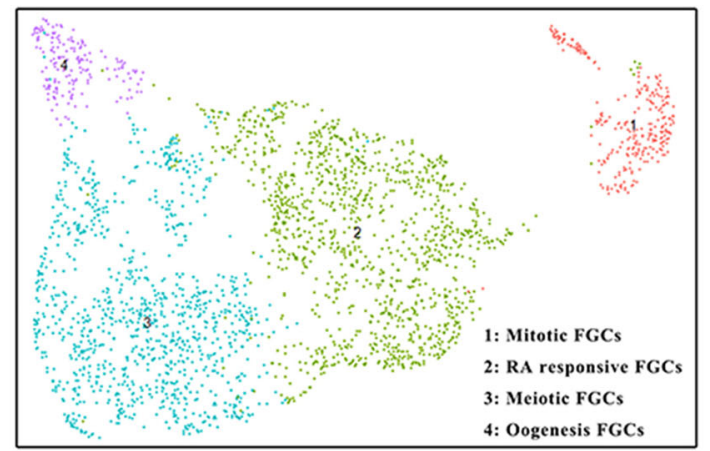

C

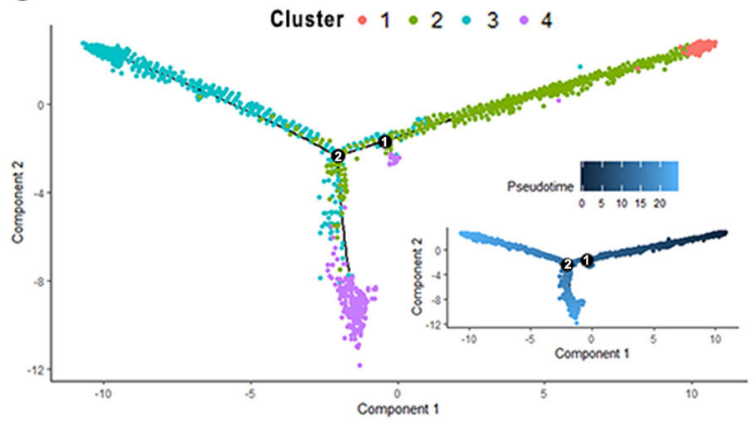

e

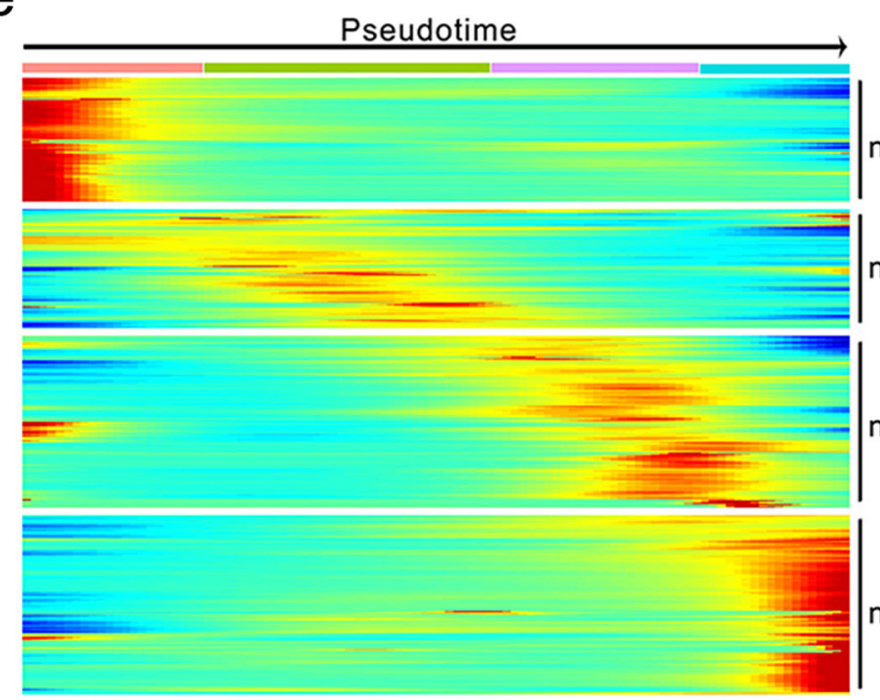

b

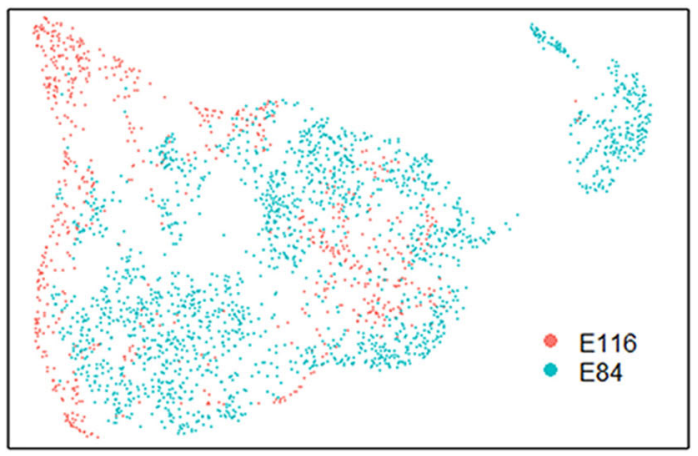

d

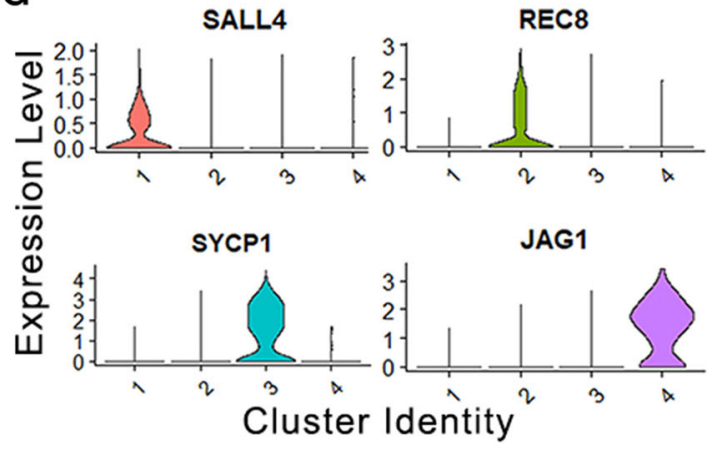

Fig. 2 Cell lineage reconstruction and identification of the germ cells. a Isolation and re-clustering of the germ cell populations at a higher resolution. The assigned clusters are consistent with that in Fig. 1b. b UMAP plot of single-cell transcriptome data with germ cells colored based on their embryonic stages. c Pseudotime analysis of germ cells. Cluster 1 represents the start of pseudotime, with cluster 4 at the end. $\mathbf{d}$ Violin plots show the expression patterns of marker genes during oogenesis. e Hierarchical clustering of the ordering genes during early oogenesis. Each row represents an ordering gene, and each column represents a single germ cell. The GO terms of clustered ordering genes are listed on the right.

STRA8, were up-regulated (Fig. 4c). Notably, the G8 and G9 at the end of the pseudotime exhibited sharply increased expression of meiotic genes, such as $S Y C P 1$ and SYCP2, while G7 and G10 highly expressed MT1X that is essential for anti-apoptosis (Fig. 4c and Supplementary Fig. S2a) ${ }^{18}$. To identify genes that were exclusively expressed in a given cell type, we performed differential gene expression analysis to identify highly variable genes 
a

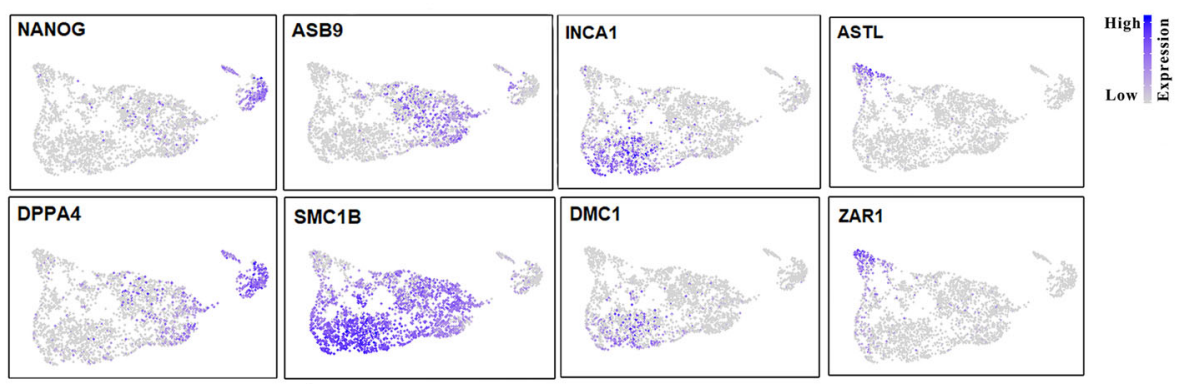

b

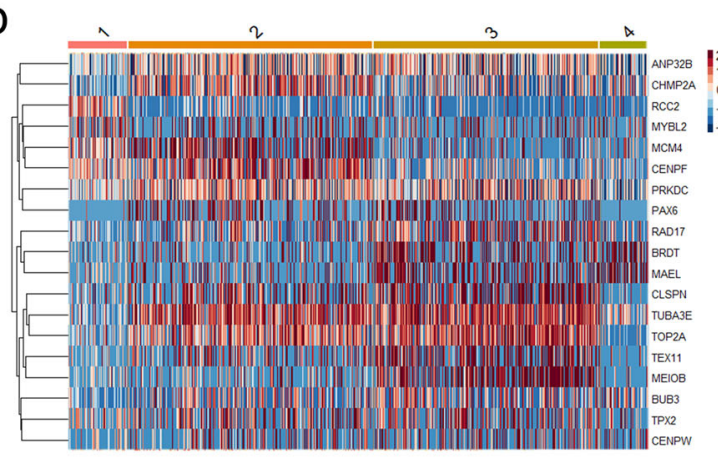

C

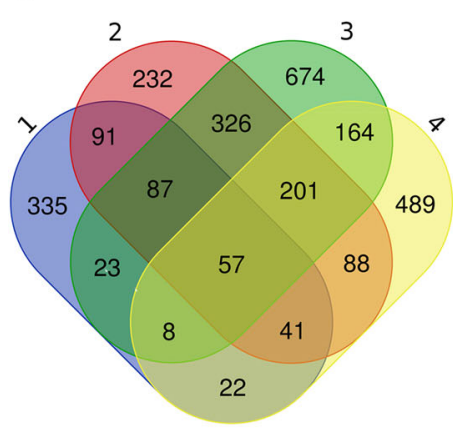

d

\section{GO terms for each cluster}
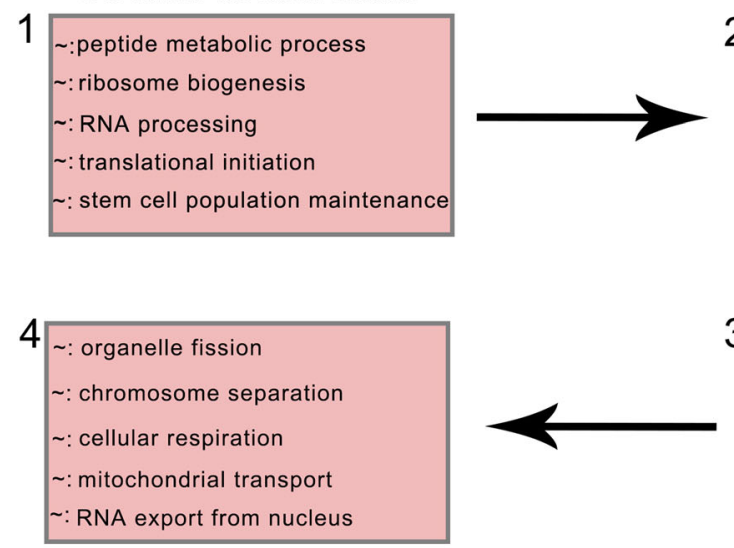

e

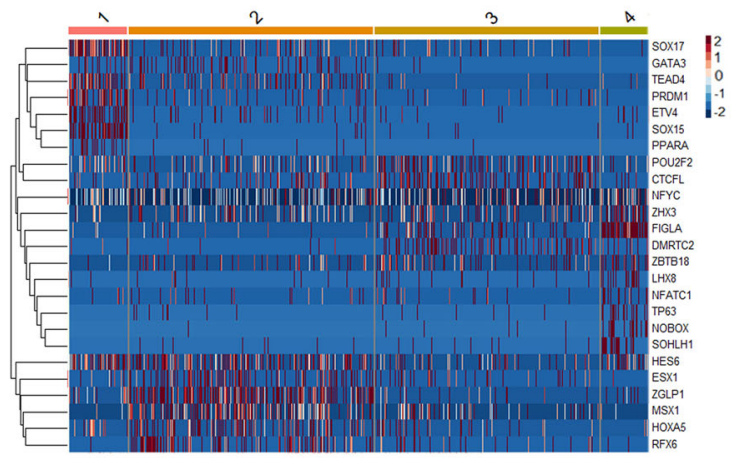

2 : mitotic cell cycle

$\sim$ :meiotic cell cycle process

$\sim:$ cell cycle phase transition

$\sim$ : oxidation-reduction process

; : mitochondrion organization

3 : meiotic cell cycle

$\sim$ : double-strand break repair

$\sim$ : chromatin organization

$\sim$ : cell cycle checkpoint

$\sim$ : male gamete generation

f

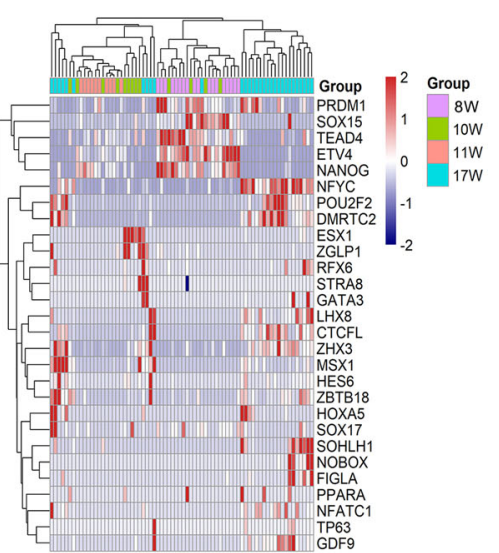

Fig. 3 (See legend on next page.) 
(see figure on previous page)

Fig. 3 Mitosis to meiosis transition and meiosis progression. a Expression patterns of germ cell development-associated genes. b Hierarchical clustering of differentially expressed mitotic and meiotic genes across female germ cell clusters (clusters 1-4). c Overlap of differentially expressed genes across germ cell clusters. d Differentially expressed genes and associated GO categories characteristic of meiosis initiation and progression, based on the four germ cell clusters. e Heatmap representing the key transcription factors across monkey germ cell clusters. $\mathbf{f}$ Hierarchical clustering of the transcription factors and human female germ cells. Each row represents a transcription factor, and each column represents a single human female germ cell.

for each cluster (Supplementary Data S4). Venn diagram shows the significant differences between G7/G10 and G8/G9 (Fig. 4d). Moreover, GO analysis on the differentially expressed genes across from G7 to G10 showed that the GO term "execution phase of apoptosis" was highly enriched in the G9 cluster (Fig. 4e), suggesting that the cells in G9 may undergo apoptosis.

To further dissect the differences between apoptotic and oogenic germ cells, we selected several marker genes that were differentially expressed among these clusters (Fig. 4f and Supplementary Fig. S2b). For example, NLRP7 is specifically expressed in germ cells that undergo oogenesis $^{19}$, but this gene is not expressed in G8 and G9, which indicates that the cells in cluster G8 and G9 are probably primed for the apoptosis process. Moreover, several genes associated with germ cell development, such as $K I T$ that is essential for germ cell survival ${ }^{20}$, also exhibit similar expression patterns with NLRP7. Furthermore, ZGLP1 was shown to induce the oogenic fate in mice ${ }^{16}$, which is absent in clusters G8 and G9 but expressed in other meiotic germ cells. In contrast, meiotic genes, such as SPO11, PRDM9, and DMC1, were specifically expressed in G8 and G9 clusters. And PRDM9-mediated H3K4me3 at hotspots could direct double-strand breaks (DSBs) fate during meiosis recombination ${ }^{21}$. Moreover, WDR87, SPDYA, and SCML1 also exhibit higher expression in G8 and G9, which may be critical for the regulation of germ cell fates (Supplementary Fig. S2b). In addition, hierarchical cluster analysis of these genes also showed that the apoptosis-associated genes, such as CSAP2, CASP9, and APAF1, were close to the meiotic genes, such as $P R D M 9, D M C 1$, and SPO11, rather than NLRP7 and KIT. Furthermore, CSAP2, CASP9, and $A P A F 1$ exhibited similar expression patterns with PRDM9, DMC1, and SPO11 in human FGCs (Fig. 4g), which further confirms that the germ cells that sharply express meiotic genes, such as SPO11, DMC1, and $P R D M 9$, may undergo apoptosis. Collectively, our findings indicate that the majority of FGCs are probably ready to undergo apoptosis, which may contribute to FGC attrition ${ }^{22}$.

\section{Identification of the granulosa cell subpopulations}

Granulosa cells are the most important somatic cells in the ovary, which markedly express $A M H R 2, F O X L 2$, and
FST genes (Fig. 5a), and play important roles in FGC development and in the formation of primordial follicles. To examine the features of granulosa cells more closely, we detected the differentially expressed genes in each cluster, and the highly variable expressed genes in granulosa cells were identified (Fig. 5b). For example, HES1, $N R 4 A 1$, and EGR3 were mainly expressed in cluster 5, while ADIRF, CBLN2, and LYPD1 exhibited peak expression in cluster 9. Subsequently, we performed principal component analysis (PCA) on the significant principal components to compare the transcriptomes of granulosa cells (Supplementary Fig. S3a). The PC1 axis had the most differences between the two samples. The E84 sample was located on the left side, whereas the E116 sample was located on the right side. The PC2 axis was dominated by differences in the cluster level within granulosa cells. However, we did not find any distinct boundary to group the granulosa cells.

To further explore the differences among granulosa cell clusters, we detected $326,120,212,110$, and 346 genes that differentially expressed across granulosa cell populations. Venn diagram shows that there are significant differences between cluster 5 and cluster 8 as well as cluster 6 and cluster 9 (Fig. 5c). This result suggests that the granulosa cells have undergone dramatic changes from E84 to E116 stages. To ascertain the features of granulosa cell subpopulations, we performed GO analysis on the differentially expressed genes in each cluster (Fig. 5d). As a result, the genes in cluster 5 were enriched in the categories of "negative regulation of apoptotic process" and "cell cycle arrest", while the genes in cluster 9 were enriched in the GO terms of "notch signaling pathway" and "tissue morphogenesis". Noticeably, certain genes in cluster 7 were enriched in the categories of "response to hormone". Collectively, these results indicated that subpopulations of granulosa cells play distinct roles in fetal ovary development.

To dissect the mechanisms that regulate granulosa cell development, we analyzed the differentially expressed genes and transcription factors across granulosa cell clusters. We found that highly variable genes like RSPO1, DUSP2, and COX4I2 exhibited peak expression specifically in the cluster 5 , cluster 7 , and cluster 9 , respectively (Fig. 5e). Noticeably, RSPO1, an activator of the WNT/ $\beta$-catenin pathway, is located upstream of the female sex 
a

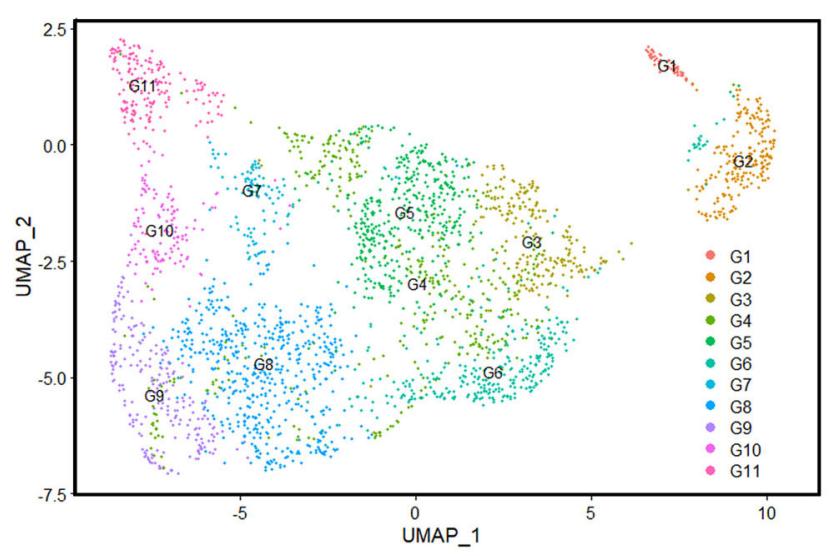

b

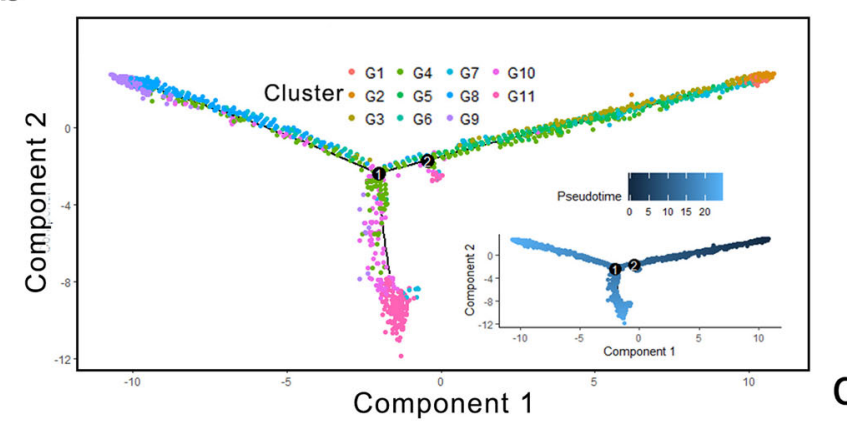

e

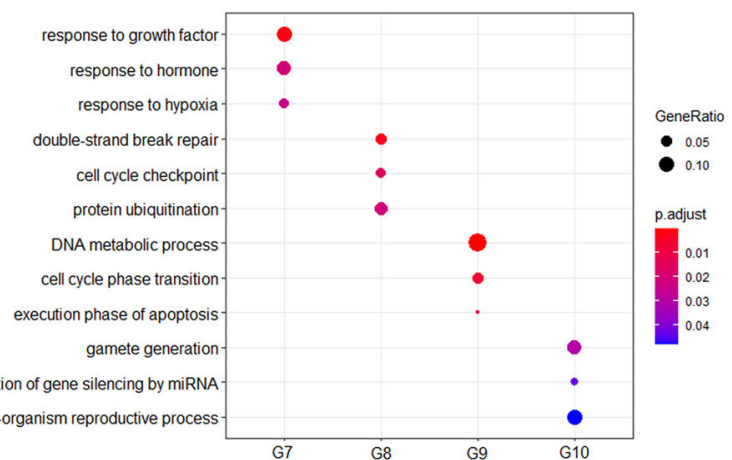

f

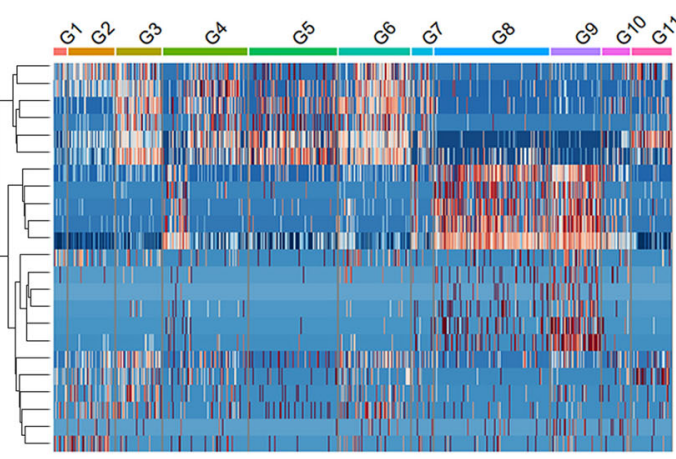

C
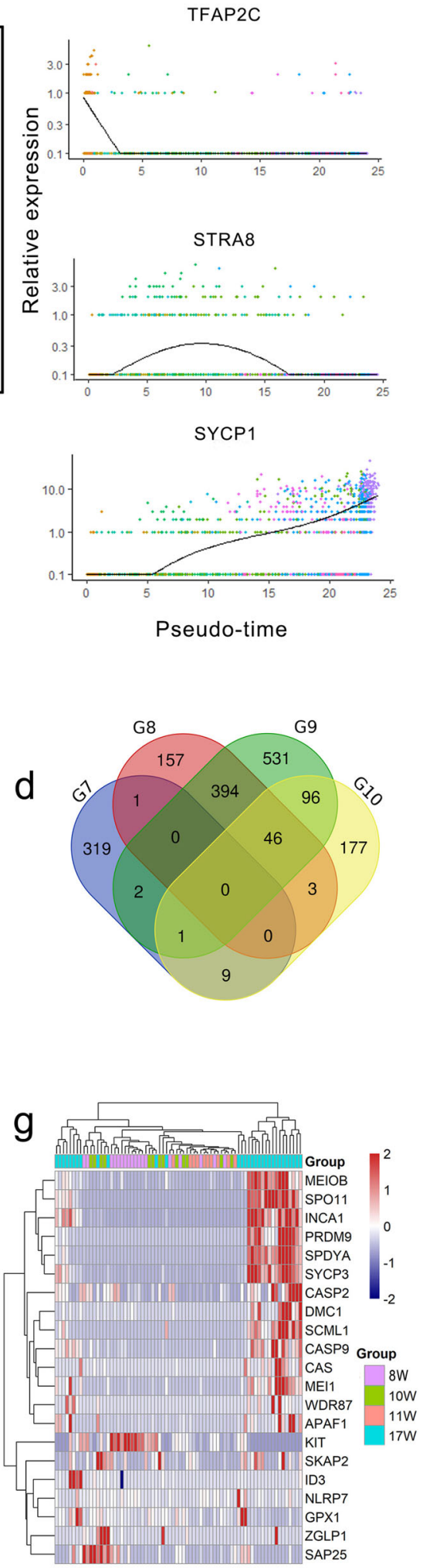

Fig. 4 (See legend on next page.) 
(see figure on previous page)

Fig. 4 Female germ cell fate determination. a Re-clustering of germ cells at a higher resolution. The 11 sub-clusters (G1-G11) were assigned and colored as indicated on the figure key. $\mathbf{b}$ Cell lineage reconstruction using monocle2. Sub-cluster G1 represents the start of pseudotime, with subcluster G11 at the end. c Dynamic expression of stage-specific genes along pseudotime. d Overlap of highly variable genes among G7-G10 subclusters. e GO analysis of differentially expressed genes in clusters G7-G10. f Heatmap representing the survival, apoptosis, and meiotic genes in monkey female germ cells. $\mathbf{g}$ Heatmap representing the survival, apoptosis, and meiotic genes in human female germ cells. Each row represents a gene, and each column represents a single human female germ cell.

determination signaling pathway, which could promote cell proliferation and ovarian differentiation ${ }^{23,24}$. In contrast, DUSP2 is a substrate for mitogen-activated protein kinases (MAPKs) ${ }^{25}$, which may play an important role in inhibiting cell proliferation. Additionally, COX4I2 is specifically expressed in cluster 9, which is involved in the modulation of oxygen affinity through hypoxia-sensing pathways, suggesting its role in the activation of primordial follicles ${ }^{26}$. Interestingly, $\mathrm{RDH} 10$ also expressed in cluster 9 (Supplementary Fig. S3b), which is essential for the production of $\mathrm{RA}^{27}$. On the other hand, transcription factors were clustered across granulosa cell subpopulations (Fig. 5f). The EGR4, ZNF597, DDIT3, MXD1, and $M A F F$ specifically expressed in cluster 5 , while $M K K$, PPARG, OSR1, HES4, THRB, and HEYL mainly expressed in cluster 8 and cluster 9. Additionally, ATF3, FOSB, and $N R 4 A 1$ displayed higher expression in cluster 5 than other populations, whereas ESR1 exhibited peak expression in cluster 7, which is consistent with the "response to hormone" category in cluster 7. Collectively, highly variable expressed transcription factors may instruct the differentiation of granulosa cells in a distinct manner.

\section{Similar characteristics between progenitor theca cells and fetal Leydig cells}

Theca cells are another important somatic cell type in the ovary, which is required for folliculogenesis. However, the origin of theca cells has not been fully demonstrated. To explore the origin of theca cells, we performed scRNAseq on the whole fetal ovary. Surprisingly, in addition to granulosa cells, there are four groups of cells that highly expressed fetal Leydig cell markers, such as DLK1, CXCL12, PDGFRA, and TCF21 (Fig. 6a). Therefore, we named these cells Leydig cells. Noticeably, Leydig cells also express NR2F2, WT1, and GLI1 (Fig. 6a), which suggested that Leydig cells may be the progenitor theca cells $^{2,28}$. To further determine the origin of theca cells, we re-clustered the Leydig cells at a higher resolution and detected the levels of theca cell marker genes. As expected, the structural theca cell marker genes PTCH1 and ACTA2 as well as endocrine theca cell marker gene CYP17A1 exhibited differentially expressed patterns across Leydig cell populations (Fig. 6b and Supplementary Fig. S3c), which indicated that progenitor theca cells exhibit the similar characteristics to fetal Leydig cells.
To further characterize theca cells, we detected the highly variable expressed genes across theca cell populations (Fig. 6c and Supplementary Fig. S4a). Obviously, cluster 11 is significantly different from the other three clusters. For example, MKI67, SGO1, CDK1, CCNA2, and $C E N P F$ were specifically expressed in cluster 11 , whereas IFI27, IFITM1, and BST2 were generally expressed in cluster 12 and cluster 13 (Fig. 6c and Supplementary Fig. S4a). Additionally, 195, 308, 71, and 192 differentially expressed genes were detected across theca cell populations. Similar to granulosa cells, theca cells exhibited distinct features between each cluster (Fig. 6d). To explore the features of theca cells, we carried out GO analysis on the differentially expressed genes in each cluster (Fig. 6e). Noticeably, the genes in cluster 13 were enriched in GO terms of "rhythmic process" and "BMP (bone morphogenic protein) singling pathway", which suggests that theca cells in cluster 13 may be involved in folliculogenesis. To dissect the mechanisms that control theca cell differentiation, we clustered the transcription factors across theca cell populations. Obviously, the transcription factors in cluster 11, such as MYBL2, CENPA, FOXM1, and $E 2 F 8$, displayed significant differences compared to that in the other three theca cell clusters (Fig. 6f). The fate of cells in cluster 11 needs to be further explored.

\section{Signaling pathways and interactions between niche and germ cells throughout fetal ovary development}

The mitosis to meiosis transition and primordial follicle formation processes are crucial steps during fetal ovary development in primates. However, the molecular mechanisms and signaling pathways that are involved in these processes have not been fully explored. Therefore, we investigated the changes in RNAs encoding signaling pathway-associated factors during fetal ovary development to provide insights into FGC development and niche-germ cell interactions.

Our sequencing data showed that the RA signaling pathway and BMP signaling pathway are involved in oogenic program initiation and meiotic progression. For the BMP signaling pathway, the ligand $B M P 2$ was highly expressed in granulosa cells, whereas the receptor $B M P R 1 B$ was expressed in both granulosa cells and FGCs. Noticeably, the targets ID1, ID2, and ID3 were expressed in FGCs in a stage-specific manner (Fig. 7a). This pattern 
a

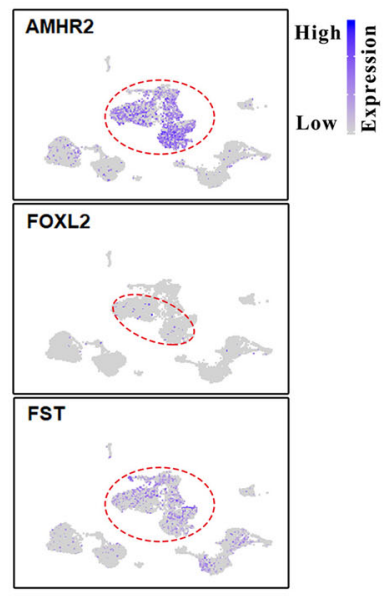

b

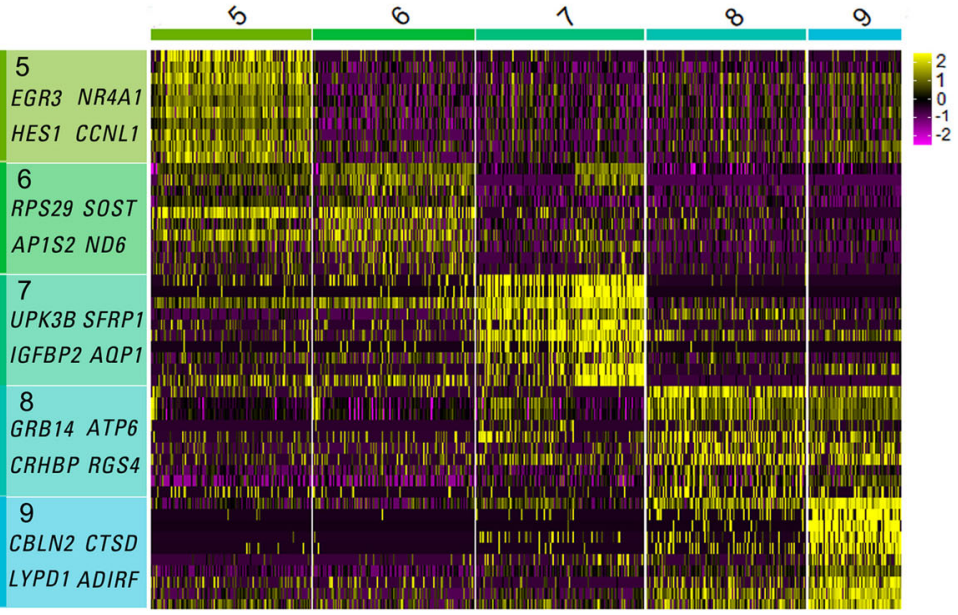

d
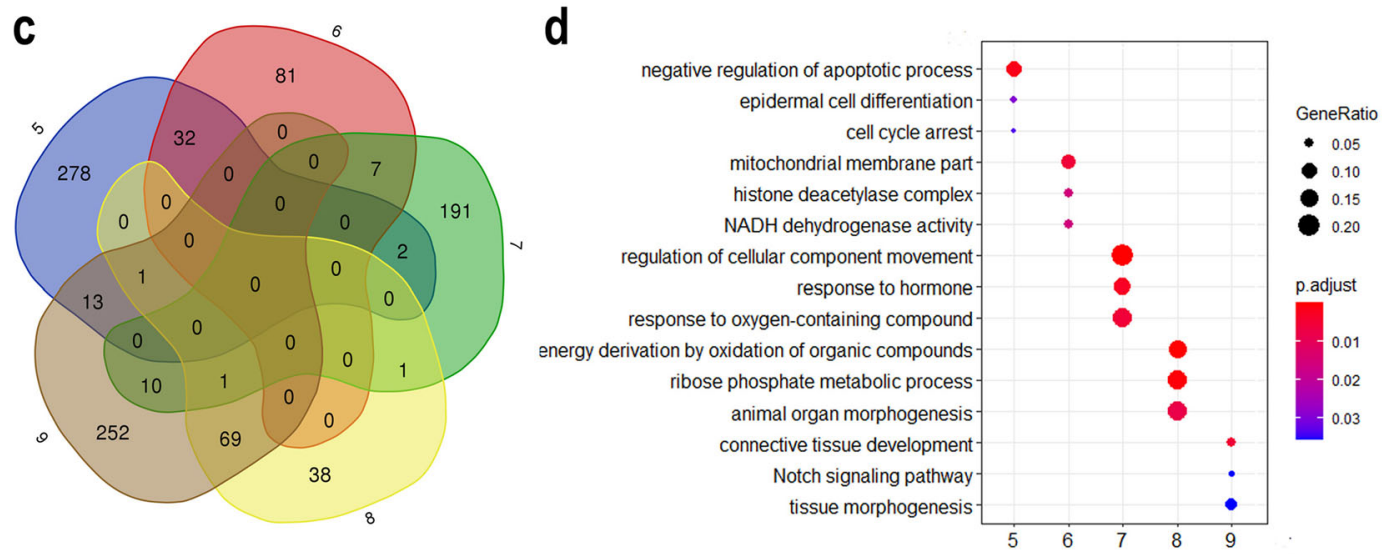

e

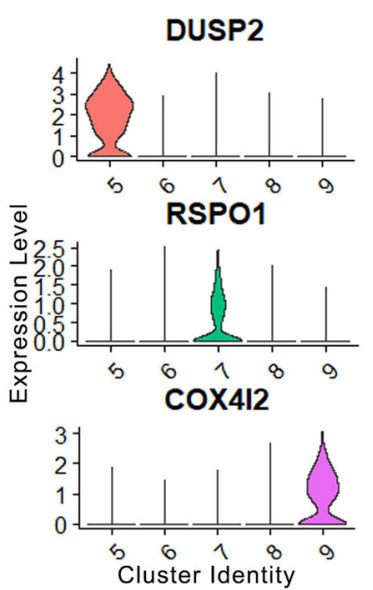

f

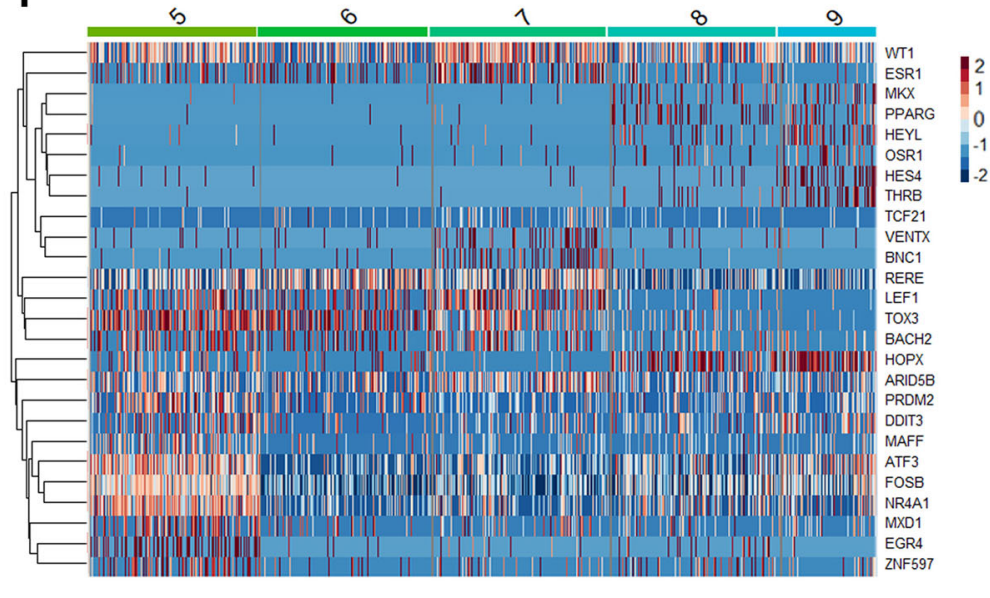

Fig. 5 Dynamic changes of granulosa cell transcriptomes. a The expression of granulosa cell marker genes, with their expression projected onto the UMAP plot. b Heatmap showing representative marker genes of granulosa cell subpopulations (clusters 5-9). c Venn diagram shows overlapping of differentially expressed genes among granulosa cell populations. $\mathbf{d}$ The enriched $\mathrm{GO}$ terms (biological processes) to the subpopulations of the granulosa cells (clusters 5-9). e Violin plots show the specific expressed genes in different granulosa cell subpopulations. $\mathbf{f}$ Heatmap representing the dynamics of transcription factors across granulosa cell populations. 

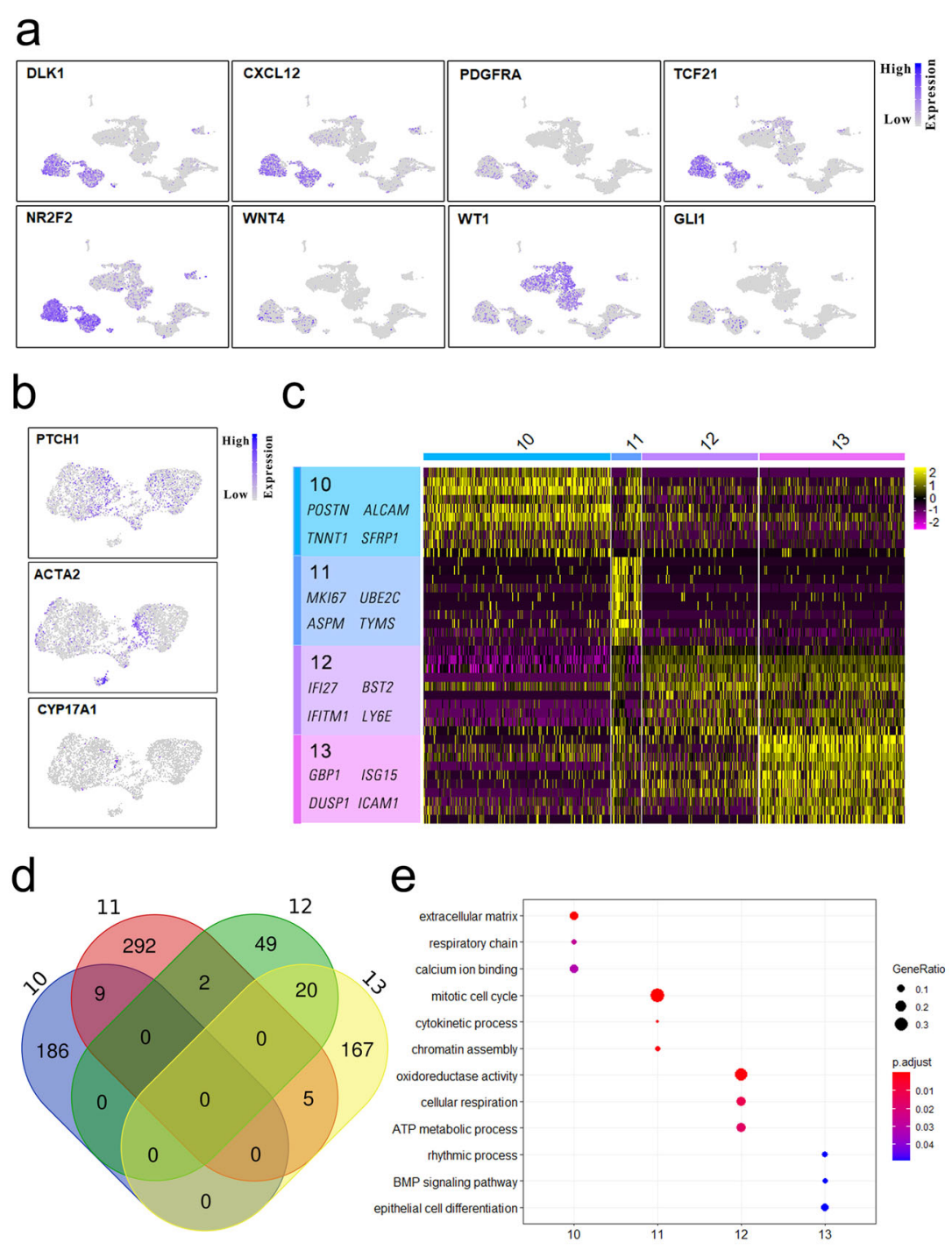

f

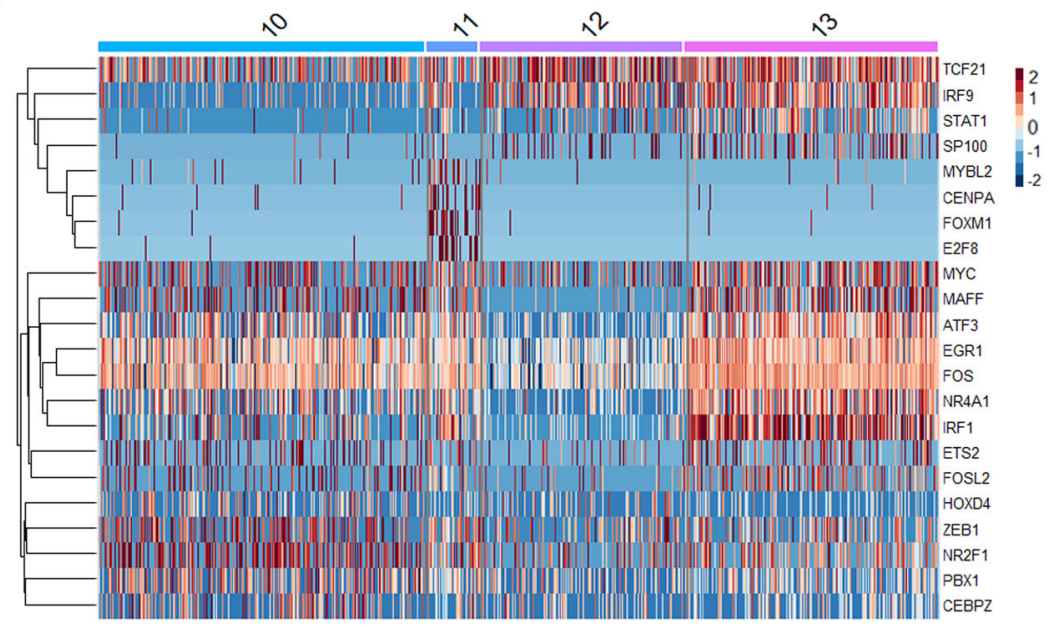

Fig. 6 Dynamic changes of transcriptome in theca cells. a UMAP cluster map showing expression of selected known marker genes for Leydig cells. $\mathbf{b}$ The expression patterns of theca cell-associated marker genes. $\mathbf{c}$ Heatmap showing representative differentially expressed genes across theca cell populations. $\mathbf{d}$ Venn diagram shows overlapping of differentially expressed genes among theca cell populations. e Top GO terms within the differentially expressed genes unique to the theca cells. $\mathbf{f}$ Hierarchical clustering of the critical transcription factors among theca cell subpopulations. 

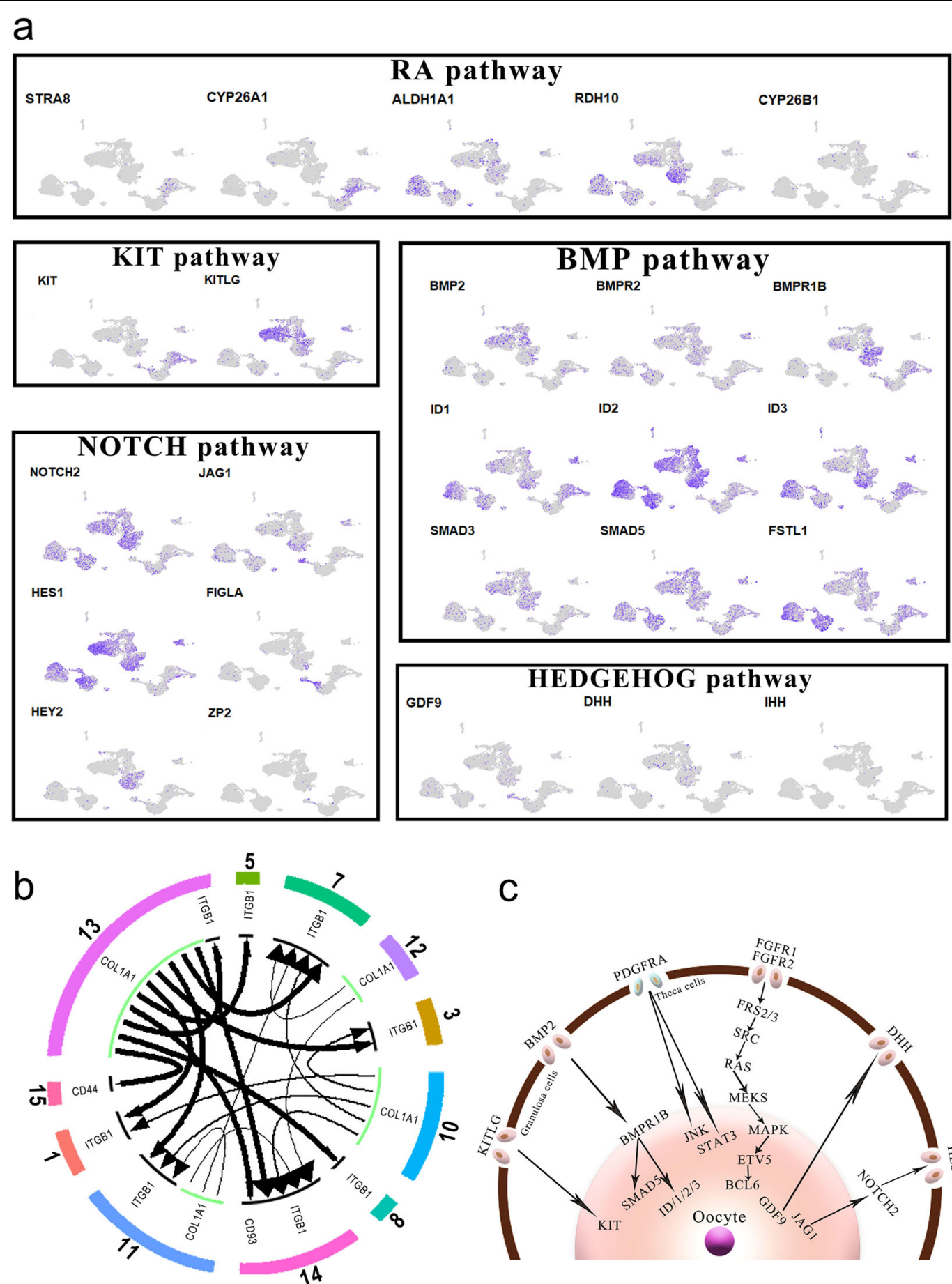

C
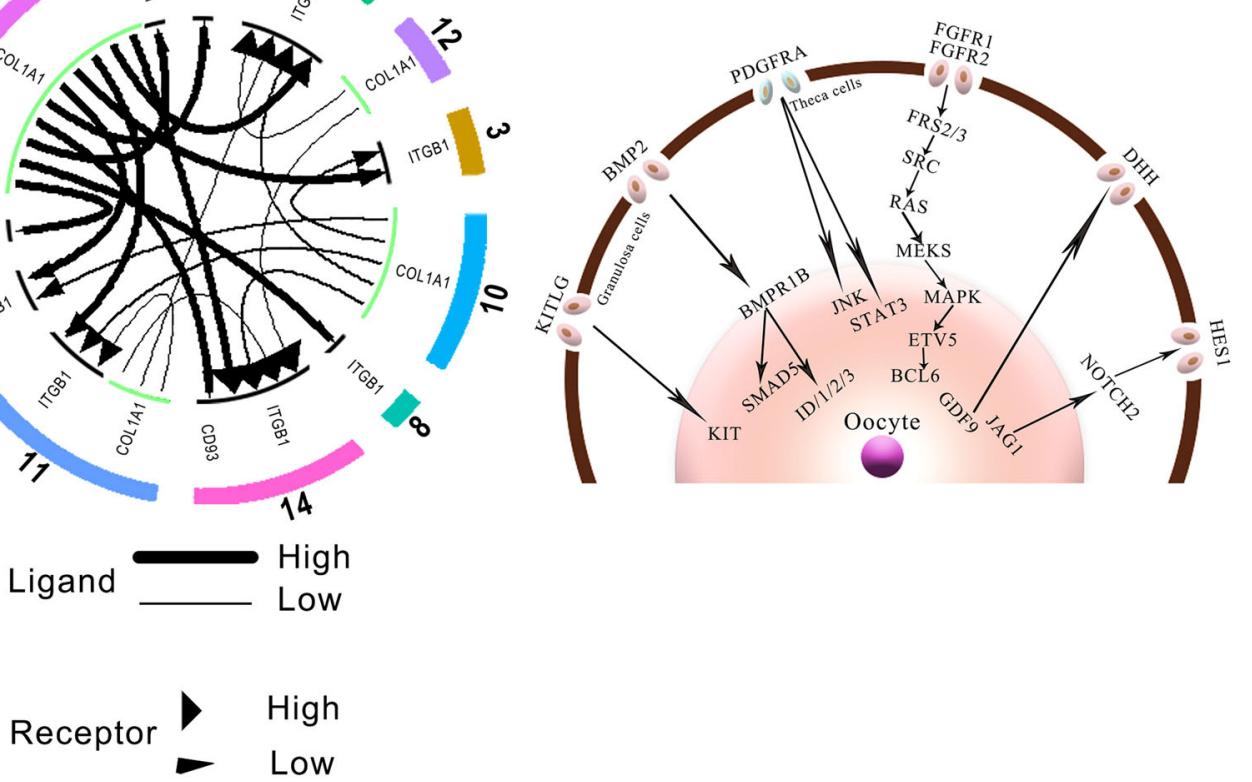

Fig. 7 Signaling pathways for niche-germline interactions. a Relative expression levels of marker genes from different key signaling pathways. b Cell-cell communication networks between different cell clusters. c Schematic summary of signaling pathways for niche-germline interactions. 
suggests that the BMP signaling pathway may play an important role in meiosis initiation and progression. Additionally, the ligand of the KIT signaling pathway (KITLG) was specifically expressed in granulosa cells, and the receptor $K I T$ was highly expressed in FGCs, which is crucial for germ cell survival $^{20}$. Interestingly, $P D G F B$ was specifically expressed in endothelial cells, and its receptors PDGFRA and PDGFRB were found in theca cells and partial granulosa cells (Supplementary Fig. S4b), suggesting that endothelial cells may indirectly affect FGC development, through the interactions with other niche cells. To further explore the crosstalk among different cell clusters within the monkey fetal ovary, we established the cell-cell communication network using iTALK package ${ }^{29}$. Notably, nearly all the ligands were derived from progenitor theca cells, and the associated receptors were enriched in other cell types (Fig. $7 \mathrm{~b}$ ), which suggests the important roles of theca cells in ovary development.

To dissect the interactions between the oocyte and granulosa cells throughout folliculogenesis, we further analyzed the expression of the factors in key signaling pathways, including NOTCH and HEDGEHOG pathways. Our results showed that the ligand JAG1 of NOTCH pathway was predominantly expressed in the oocytes at the oogenesis phase, whereas the receptor $\mathrm{NOTCH} 2$ as well as the downstream target gene HES1 were highly expressed in granulosa cells and progenitor theca cells (Fig. 7a). Next, we investigated the key components of the HEDGEHOG signaling pathway in the oocytes and somatic cells. GDF9 exhibited a high expression level in the oogenesis phase FGCs, which could promote the production of Desert hedgehog and Indian hedgehog in granulosa cells. Moreover, the receptor PTCH1 as well as downstream signaling components GLI1 of the HEDGEHOG pathway were highly expressed in theca cells (Fig. 7a, c), indicating that the HEDGEHOG pathway may play critical roles in the specification of theca cells. Taken together, our results demonstrate that reciprocally interacting signaling pathways control fetal ovary development in a stage-specific manner.

\section{Discussion}

Fetal ovary development is a complex process in primates, and a full understanding of its regulation requires the integration of multiple data collected from various cell types in the ovary. Here, we performed scRNA-seq analysis of all cells within the fetal ovaries at two different developmental stages to provide new insights into the regulation of fetal ovary development in primates. In this study, we have identified four clusters of FGCs: mitotic phase, RA-responsive phase, meiotic phase, and oogenesis phase. Moreover, the niche cells, such as granulosa cells, theca cells, endothelial cells, and macrophages, were also identified. Furthermore, our work revealed unique characteristics in transcriptional profiles and reciprocal interactions between germ cells and niche cells in each developmental stage. These identified cell types and stagespecific expressed genes in fetal ovaries may offer a valuable information for future functional studies.

FGCs undergo meiosis initiation, primordial follicle formation, and apoptosis. Here, we reconstructed the developmental trajectory of FGCs, which revealed two waves of oogenesis. Noticeably, the first minor oogenesis occurs following the RA-responsive stage, which could further undergo growth and development in the medulla ${ }^{12}$. However, these follicles have to undergo atresia due to lacking hormone stimulation in the prepuberty ovary, and cannot contribute to fertility. On the other hand, the second major oogenesis occurs in the late meiotic prophase accompanied with the apoptosis of the vast majority of germ cells. The germ cells in the second oogenesis will form primordial follicles and stay dormant in the ovarian cortex until puberty ${ }^{11}$. After puberty, the primordial follicles are recruited regularly to generate mature oocytes under the stimulation of gonadotropin hormones, which contributes to the life span of female reproduction.

The mitosis to meiosis transition is a critical step in the oogenic program; however, the mechanisms for the regulation of meiosis initiation have not been fully demonstrated. Previous studies have shown that RA induces the expression of STRA8, which could further switch on the meiotic genes, such as $S Y C P 3$, and repress the early PGC program to promote meiosis initiation. RA enhances this pathway through retinoic acid receptor (RAR)-mediated transcriptional regulation; however, after ablation of RARcoding genes, the FGCs robustly expressed meiotic genes, such as STRA8, REC8, and SYCP3, showing that RARs are actually dispensable for meiosis initiation ${ }^{30}$. Moreover, recent studies indicate that $Z g l p 1$ is partially overlapped with Nanog in mice, which is sufficient to induce the oogenic fate and meiosis entry, whereas RA augments the ZGLP1-activated oogenic program and meiosis initiation $^{16,31}$. In our study, ZGLP1 is mainly expressed in germ cells at the RA-responsive stage, which is not overlapped with NANOG in monkey FGCs, indicating that ZGLP1 is essential for meiosis entry but not for activating the oogenic fate in primates. Moreover, ZGLP1 is also expressed in the oogenesis phase germ cells, suggesting its roles in subsequent oogenesis.

During the second wave of oogenesis, the germ cells are surrounded by somatic cells to form primordial follicles, while the vast majority of germ cells undergo apoptosis. Reconstructed germ cell lineage revealed that C8 and C9 are likely to undergo apoptosis, while other germ cells maintain survival and undergo oogenesis. NLRP7 is generally expressed in all stages of oogenesis, and KIT is crucial for the survival of germ cells ${ }^{19,20}$. However, NLRP7 
and KIT were not expressed in the C8 and C9. Noticeably, several meiosis-associated genes, such as SPO11, PRDM9, $D M C 1$, and INCA1, exhibited peak expression in C8 and C9. Recent studies have shown that PRDM9-mediated H3K4me3 could guide SPO11 targeting to induce DSBs ${ }^{32}$. Moreover, earlier formed DSBs occupy more open chromatin and are much more competent to proceed to a crossover fate, whereas later formed DSBs are likely to proceed to a non-crossover fate ${ }^{21}$. In this study, PRDM9 and $\mathrm{SPO} 11$ specifically expressed in $\mathrm{C} 8$ and $\mathrm{C} 9$, whose DSBs are formed late causing their non-crossovers fate, which may be the cause for apoptosis of germ cells in C8 and C9.

Ovary and testis have the same developmental origin: the bipotential gonads that are composed of multipotent somatic progenitor cells. After sex determination, the somatic progenitor cells differentiate into Sertoli cells and Leydig cells in male gonads, or granulosa cells and theca cells in female gonads. Our data revealed five clusters of granulosa cells that displayed distinct features in late stage ovaries. In addition, we also provide several new insights into theca cell development, most importantly into the origin of theca cells. Apart from the somatic progenitor cells that differentiated into granulosa cells, the remaining somatic progenitor cells exhibited peak expression of NR2F2, which acquire a steroidogenic precursor fate by progressively expressing CYP17A1, PTCH1, and ACTA2. Interestingly, these cells also exhibited the Leydig cell features, which highly expressed Leydig cell marker genes, such as DLK1, $T C F 21$, and CXCL12. In contrast to $S R Y$ that is essential for male sex determination, NR2F2 has been shown to control female sex determination through eliminating Wolffian ducts in female embryos ${ }^{33}$. Moreover, $N R 2 F 2$, a lineagespecific transcription factor, plays important roles in celltype specification and cell fate maintenance ${ }^{34}$. Therefore, we speculated that progenitor theca cells exhibit similar features to fetal Leydig cells, and the features of Leydig cells are eliminated gradually through the development of the ovary dependent on NR2F2.

A major area of current interest involves niche-germline communications that coordinately and reciprocally regulate fetal ovary development, but knowledge on how the signaling pathways interact remains limited. In this study, we have identified many ligands and receptors that are derived from the reciprocal compartments. For example, we found that the BMP signaling pathway was activated in FGCs via granulosa cells-driven mechanisms. BMP2, the ligands for the BMP signaling pathway, was specifically expressed in granulosa cells, while its receptor, BMPR1B, and downstream effector, ZGLP1, were expressed in FGCs. Our data concur that the BMP-ZGLP1 pathway could also activate the oogenic program in primates. Moreover, the typical RA pathway contributes to oogenic program maturation and
PGC program repression. In contrast, $\mathrm{NOTCH}$ and HEDGEHOG pathways were activated in niche cells. For instance, the ligand $J A G 1$ of the $\mathrm{NOTCH}$ pathway was specifically expressed in the oocytes, while its receptor $\mathrm{NOTCH} 2$ as well as the downstream target gene HES1 were highly expressed in granulosa cells and progenitor theca cells. In this study, we have identified several pathways that may govern the fetal ovary development in primates, which provides valuable information for the improvement of in vitro culture of gametes.

In summary, this work provides new insights into the crucial features of monkey fetal ovaries especially in the late embryonic stages. Our study paves the way for understanding the molecular regulation of fetal FGC development, including meiosis initiation, primordial follicle formation, and apoptosis of germ cells. It also lays a solid foundation for the theca cell origin identity. More importantly, the reciprocal relationship between the signaling pathways of FGCs and their niche cells will provide a valuable resource for further optimizing and improving the efficiency of germ cell differentiation in vitro.

\section{Materials and methods \\ Animal ethics statements}

Fetal female cynomolgus monkeys (Macaca fascicularis) were selected for this study. The use and care of animals complied with the guideline of the Animal Advisory Committee at the Institute of Neuroscience, Chinese Academy of Sciences. The ethics application entitled "Construction of cynomolgus monkey model based on somatic cell nuclear transfer" (\#ION-2018002R01) was approved by the Institute of Neuroscience, Chinese Academy of Sciences.

\section{Sample collection}

The ovary samples for scRNA-seq were from two fetal female Macaca fascicularis (embryonic days 84 and 116). For each single-cell sequencing experiment, two ovaries were washed twice in $1 \times$ PBS, and subjected to a standard digestion procedure through Tumor Dissociation Kit, human (MiltenyiBiotec \#130-095-929). First, the ovaries were cut into small pieces of $2-4 \mathrm{~mm}$, and transferred into the gentleMACS C Tube containing the enzyme mix (4.7 mL DMEM, $200 \mu \mathrm{L}$ Enzyme H, $100 \mu \mathrm{L}$ Enzyme R, and $25 \mu \mathrm{L}$ Enzyme A). Then, the $\mathrm{C}$ tube was attached onto the sleeve of the gentleMACSdissociator. After termination of the program, the $C$ tube was detached from the gentleMACSDissociator. The sample was incubated for $30 \mathrm{~min}$ at $37^{\circ} \mathrm{C}$ under continuous rotation using the MACSmix Tube Rotator. Next, the $\mathrm{C}$ tube was attached onto the sleeve of the gentleMACSdissociator, and then subjected to a short centrifugation step to collect the sample material at the bottom of the tube. The sample was resuspended and the cell suspension was applied to a 
MACS SmartStrainer $(70 \mu \mathrm{m})$ placed on a $50 \mathrm{~mL}$ tube and washed in a cell MACS SmartStrainer $(70 \mu \mathrm{m})$ with $20 \mathrm{~mL}$ DMEM. Finally, the cell suspension was centrifuged at $300 \times g$ for $7 \mathrm{~min}$, the supernatant was completely aspirated and cells were resuspended as required for further applications.

\section{Construction of single-cell RNA libraries and sequencing}

Single-cell suspensions of ovary cells were captured on a $10 \times$ Chromium system (10× Genomics). Then, single-cell mRNA libraries were generated using the single-cell $3^{\prime}$ reagent V3 kits according to the manufacturer's protocol. About 16,000 cells were added to each channel with a targeted cell recovery estimate of 8000 cells $(10,000$ for E84 monkey and 12,947 for E116 monkey). After generation of GEMs, reverse transcription reactions were barcoded using a unique molecular identifier (UMI) and cDNA libraries were then amplified by PCR with appropriate cycles. Subsequently, the amplified cDNA libraries were fragmented and then sequenced on an Illumina NovaSeq 6000 (Illumina, San Diego).

\section{ScRNA-seq data processing and analysis}

The Cell Ranger software (version 3.0.2) was used to perform sample demultiplexing, reads mapping and barcode processing to generate a matrix of gene counts versus cells. Briefly, the raw BCL files generated by Illumina NovaSeq 6000 sequencing were demultiplexed into fastq files through the Cell Ranger $m k f a s t q$ pipeline. Next, the fastq files were processed using the Cell Ranger count pipeline to map high-quality reads to the monkey reference genome (Macaca_fascicularis_5.0). Aligned reads were further filtered for valid cell barcodes and UMIs to produce a count matrix.

Then, count matrix was imported into the $\mathrm{R}$ package Seurat ${ }^{8}$ and quality control was performed to remove outlier cells and genes. Cells with 200-3000 detected genes were retained. Genes were retained in the data if they were expressed in $\geq 3$ cells. After applying these quality control criteria, 12471 cells and 18446 genes remained for downstream analyses. Additional normalization was performed in Seurat on the filtered matrix to obtain the normalized count. Highly variable genes across single cells were identified and PCA was performed to reduce the dimensionality on the top 18 principal components. Then, cells were clustered at a resolution of 0.6 and visualized in two-dimensions using UMAP9

\section{Pseudotime analysis of single-cell transcriptomes}

Germ cell lineage trajectories were constructed according to the procedure recommended in the Monocle2 documentation (http://cole-trapnell-lab.github.io/ monocle-release/docs ${ }^{10}$. Cluster 1 (mitotic PGCs) was used as start, cluster 2 (meiotic PGCs) as middle, and cluster 4 (early activated oocytes) as the end of pseudotime. Briefly, the top differentially expressed genes were selected as "ordering genes" to recover lineage trajectories in Monocle2 using default parameters. After pseudotime time was determined, differentially expressed genes were clustered to verify the fidelity of lineage trajectories. Additionally, the expression patterns of key germ cell markers across pseudospace were visualized through the function of plot_genes_in_pseudotime in Monocle2.

\section{GO analysis}

Enrichment analysis for highly variable genes detected per cluster was conducted using ClusterProfiler R package $^{35}$. Symbol gene IDs were translated into Entre IDs through bitr function. The analysis of the enrichment of differentially expressed genes was performed and a corrected $P$ value $\leq 0.05$ was considered to indicate significant gene enrichment.

\section{Transcription factor analysis}

To show the dynamics of transcription factors during FGC and somatic cell development, we downloaded all 1426 monkey transcription factors from Animal TFDB 3.0 (http://bioinfo.life.hust.edu.cn/AnimalTFDB). Then, the differentially expressed genes across 15 cell clusters and monkey transcription factors were intersected to obtain differentially expressed transcription factors, which were further visualized using heatmap.

\section{Cell-cell communication analysis}

Cell-cell communication was analyzed using iTALK package $^{29}$. The input data were Seurat object, and the ligand-receptor pairs were detected from top $50 \%$ highly expressed genes. The communication types mainly included growth factors, cytokines, and checkpoint. The network plot was visualized using LRPlot function.

\section{Transcriptome analysis of human FGCs}

The scRNA-seq data were downloaded from NCBI Gene Expression Omnibus (GEO) database ${ }^{5}$, and the associated accession number was GSE63818. The gene expression matrix was established through merging the data of 72 single cells ranging from 8 to 17 weeks (Supplementary Data S5). The dynamics of transcription factors and critical genes during FGC development were visualized using heatmap with hierarchical clustering of columns and rows.

\section{Acknowledgements}

We appreciate help from the members of the Sun laboratory for comments during preparation of the manuscript. We thank Fei-Yang Wang from State Key Laboratory of Stem Cell and Reproductive Biology, Institute of Zoology, Chinese Academy of Sciences for assistance with bioinformatic analysis. This study was funded by the National R\&D Program of China (2018YFA0107701): the National Natural Science Foundation of China (31801245); the Strategic Priority Research Program of the Chinese Academy of Sciences (XDB32060100), 
the Shanghai Municipal Science and Technology Major Project (2018SHZDZX05), the Shanghai Municipal Government Bureau of Science and Technology (18JC1410100), the National Key Research and Development Program of China (2018YFC1003000), and the National Natural Science Foundation of China Grant (31825018).

\section{Author details}

${ }^{1}$ State Key Laboratory of Stem Cell and Reproductive Biology, Institute of Zoology, Chinese Academy of Sciences, 100101 Beijing, China. ${ }^{2}$ University of Chinese Academy of Sciences, 100049 Beijing, China. ${ }^{3}$ Institute of Neuroscience, CAS Center for Excellence in Brain Science and Intelligence Technology, State Key Laboratory of Neuroscience, CAS Key Laboratory of Primate Neurobiology, Chinese Academy of Sciences, 200031 Shanghai, China. ${ }^{4}$ Fertility Preservation Lab, Reproductive Medicine Center, Guangdong Second Provincial General Hospital, 510317 Guangzhou, Guangdong, China. ${ }^{5}$ Department of Reproductive Medicine Center, Third Affiliated Hospital of Guangzhou Medical University, 510150 Guangzhou, Guangdong, China. ${ }^{6}$ Department of Veterinary Pathobiology, University of Missouri, Columbia, MO 65211, USA

\section{Author contributions}

Q.-Y.S. and Q.S. conceived the project and designed the experiments. Z.-H.Z. conducted the experiment and performed the data analysis. Sample collection was led by C.-Y.L and Y.W. T.-G.M., W.-B.L., A.L., Y.-J.C., and Y.H. provided the technical support. Q.-Y.S. and Z.-H.Z. wrote the paper. Q.-Y.S., H.S., and Z.-B.W. edited the manuscript. All authors read and approved the final manuscript.

\section{Data availability}

The high-throughput sequencing data in this study have been deposited in the Gene Expression Omnibus (GEO) database under accession number GSE149629. To review GEO accession GSE149629: Go to https://www.ncbi.nlm. nih.gov/geo/query/acc.cgi?acc=GSE149629. Enter token exkjqgaibhyfhqt into the box.

\section{Conflict of interest}

The authors declare that they have no conflict of interest.

\section{Publisher's note}

Springer Nature remains neutral with regard to jurisdictional claims in published maps and institutional affiliations.

Supplementary Information accompanies the paper at (https://doi.org/ 10.1038/s41421-020-00219-0).

Received: 19 June 2020 Accepted: 22 September 2020

Published online: 29 December 2020

\section{References}

1. Bullejos, M. \& Koopman, P. Germ cells enter meiosis in a rostro-caudal wave during development of the mouse ovary. Mol. Reprod. Dev. 68, 422-428 (2004).

2. Stévant, l. et al. Single-cell transcriptomics of the mouse gonadal soma reveals the establishment of sexual dimorphism in distinct cell lineages. https://www. biorxiv.org/content/10.1101/410407v1.full (2018).

3. Evans, E. P., Ford, C. E. \& Lyon, M. F. Direct evidence of the capacity of the XY germ cell in the mouse to become an oocyte. Nature 267, 430-431 (1977).

4. Li, L. et al. Single-cell RNA-seq analysis maps development of human germline cells and gonadal niche interactions. Cell Stem Cell 20, 891-892 (2017).

5. Guo, F. et al. The transcriptome and DNA methylome landscapes of human primordial germ cells. Cell 161, 1437-1452 (2015).

6. Mayère, C. et al. Single-cell transcriptomic reveals temporal dynamics of critical regulators of germ cell fate during mouse sex determination. https://www. biorxiv.org/content/10.1101/747279v1 (2019).

7. Magnusdottir, E. et al. A tripartite transcription factor network regulates primordial germ cell specification in mice. Nat. Cell Biol. 15, 905-915 (2013).
8. Butler, A., Hoffman, P., Smibert, P., Papalexi, E. \& Satija, R. Integrating single-cell transcriptomic data across different conditions, technologies, and species. Nat. Biotechnol. 36, 411-420 (2018).

9. Mclnnes, L., Healy, J. \& Melville, J. UMAP: Uniform Manifold Approximation and Projection for dimension reduction. https://arxiv.org/abs/1802.03426 (2018).

10. Qiu, X. et al. Reversed graph embedding resolves complex single-cell trajectories. Nat. Methods 14, 979-982 (2017).

11. Mork, L. et al. Temporal differences in granulosa cell specification in the ovary reflect distinct follicle fates in mice. Biol. Reprod. 86, 37 (2012).

12. Byskov, A. G. et al. No evidence for the presence of oogonia in the human ovary after their final clearance during the first two years of life. Hum. Reprod. 26, 2129-2139 (2011).

13. Hayashi, K., Ohta, H., Kurimoto, K., Aramaki, S. \& Saitou, M. Reconstitution of the mouse germ cell specification pathway in culture by pluripotent stem cells. Cell 146, 519-532 (2011).

14. Ohinata, Y. et al. A signaling principle for the specification of the germ cell lineage in mice. Cell 137, 571-584 (2009).

15. Le Bouffant, R. et al. Msx1 and Msx2 promote meiosis initiation. Development 138, 5393-5402 (2011).

16. Nagaoka, S. I. et al. ZGLP1 is a determinant for the oogenic fate in mice. Science 367, eaaw4115 (2020).

17. Jan, S. Z. et al. Unraveling transcriptome dynamics in human spermatogenesis. Development 144, 3659-3673 (2017).

18. Peng, B., Gu, Y., Xiong, Y., Zheng, G. \& He, Z. Microarray-assisted pathway analysis identifies MT1X \&NFkappaB as mediators of TCRP1-associated resistance to cisplatin in oral squamous cell carcinoma. PLOS ONE 7, e51413 (2012).

19. Amoushahi, M., Sunde, L. \& Lykke-Hartmann, K. The pivotal roles of the NODlike receptors with a PYD domain, NLRPs, in oocytes and early embryo development. Biol. Reprod. 101, 284-296 (2019).

20. Kissel, $\mathrm{H}$. et al. Point mutation in kit receptor tyrosine kinase reveals essential roles for kit signaling in spermatogenesis and oogenesis without affecting other kit responses. EMBO J. 19, 1312-1326 (2000).

21. Chen, Y. et al. Refined spatial temporal epigenomic profiling reveals intrinsic connection between PRDM9-mediated H3K4me3 and the fate of doublestranded breaks. Cell Res. 30, 256-268 (2020).

22. Findlay, J. K., Hutt, K. J., Hickey, M. \& Anderson, R. A. How is the number of primordial follicles in the ovarian reserve established? Biol. Reprod. 93, 111 (2015).

23. Chassot, A. A. et al. WNT4 and RSPO1 together are required for cell proliferation in the early mouse gonad. Development 139, 4461-4472 (2012).

24. Chassot, A. A. et al. RSPO1/beta-catenin signaling pathway regulates oogonia differentiation and entry into meiosis in the mouse fetal ovary. PLOS ONE 6, e25641 (2011).

25. Perander, M. et al. Regulation of atypical MAP kinases ERK3 and ERK4 by the phosphatase DUSP2. Sci. Rep. 7, 43471 (2017).

26. PajueloReguera, D. et al. Cytochrome c oxidase subunit 4 isoform exchange results in modulation of oxygen affinity. Cells $\mathbf{9 , 4 4 3}$ (2020).

27. Metzler, M. A. et al. RDH10-mediated retinol metabolism and RARalphamediated retinoic acid signaling are required for submandibular salivary gland initiation. Development 145, dev.164822 (2018).

28. Liu, C., Peng, J., Matzuk, M. M. \& Yao, H. H. Lineage specification of ovarian theca cells requires multicellular interactions via oocyte and granulosa cells. Nat. Commun. 6, 6934 (2015).

29. Wang, $Y$. et al. iTALK: an R package to characterize and Illustrate intercellular communication. https://www.biorxiv.org/content/10.1101/507871v1 (2019).

30. Vernet, $\mathrm{N}$. et al. Meiosis initiates in the fetal ovary of mice lacking all retinoic acid receptor isotypes. Sci. Adv. 6, eaaz1139 (2020).

31. Zhao, Z.-H. et al. Single cell RNA sequencing reveals the landscape of early female germ cell development. FASEB J. (2020).

32. Paiano, J. et al. ATM and PRDM9 regulate SPO11-bound recombination intermediates during meiosis. Nat. Commun. 11, 857 (2020).

33. Zhao, F. et al. Elimination of the male reproductive tract in the female embryo is promoted by COUP-TFII in mice. Science 357, 717-720 (2017).

34. Sissaoui, S. et al. Genomic characterization of endothelial enhancers reveals a multifunctional role for NR2F2 in regulation of arteriovenous gene expression. Circ. Res. 126, 875-888 (2020).

35. Yu, G., Wang, L. G., Han, Y. \& He, Q. Y. clusterProfiler: an R package for comparing biological themes among gene clusters. OMICS 16, 284-287 (2012). 